\title{
Cold dust in a selected sample of nearby galaxies
}

\section{The interacting galaxy NGC 4631}

\author{
M. Dumke ${ }^{1,2}$, M. Krause ${ }^{1}$, and R. Wielebinski ${ }^{1}$ \\ 1 Max-Planck-Institut für Radioastronomie, Auf dem Hügel 69, 53121 Bonn, Germany \\ 2 SMTO, Steward Observatory, The University of Arizona, 933 N. Cherry Avenue, Tucson, Arizona 85721, USA
}

Received 14 April 2003 / Accepted 17 October 2003

\begin{abstract}
We have observed the continuum emission of the interacting galaxy NGC 4631 at $\lambda \lambda 870 \mu \mathrm{m}$ and $1.23 \mathrm{~mm}$ using the Heinrich-Hertz-Telescope on Mt. Graham and the IRAM 30-m telescope on Pico Veleta. We have obtained fully sampled maps which cover the optical emission out to a radius of about $7^{\prime}$ at both wavelengths. For a detailed analysis, we carefully subtracted the line contributions and synchrotron and free-free emission from the data, which added up to $6 \%$ at $1.23 \mathrm{~mm}$ and $10 \%$ at $0.87 \mathrm{~mm}$. We combined the flux densities with FIR data to obtain dust spectra and calculate dust temperatures, absorption cross sections, and masses. Assuming a "standard" dust model, which consists of two populations of big grains at moderate and warm temperatures, we obtained temperatures of $18 \mathrm{~K}$ and $50 \mathrm{~K}$ for the both components. However, such a model suffers from an excess of the radiation at $\lambda 1.23 \mathrm{~mm}$, and the dust absorption cross section seems to be enhanced by a factor 3 compared to previous results and theoretical expectations. At large galactocentric radii, where the galaxy shows disturbances as a result of gravitational interaction, this effect seems to be even stronger. Some possibilities to resolve these problems are discussed. The data could be explained by a very cold dust component at a temperature of $4-6 \mathrm{~K}$, an increased abundance of very small grains, or a component of grains with unusual optical properties. We favour the latter possibility, since the first two lead to inconsistencies
\end{abstract}

Key words. galaxies: ISM - radio continuum: galaxies - galaxies: individual: NGC 4631

\section{Introduction}

The efficiency and time-scale of star-formation processes in galaxies depend strongly on the amount, distribution, and composition of the interstellar matter. One component of the ISM whose properties are still not well known is interstellar dust.

Since most of the dust in galaxies is cold, with temperatures of $<20 \mathrm{~K}$, infrared observatories like IRAS are almost blind for the gross amount of dust, and can only observe the warm dust component. The major fraction of the interstellar dust radiates mainly in the sub-mm range, at wavelengths $>100 \mu \mathrm{m}$. This emission was hardly accessible in the past, due to the lack of good sub-mm telescopes at sites with sufficiently good atmospheric conditions. This situation has improved in the last few years: instruments like the bolometer arrays installed at the 30-m telescope on Pico Veleta or - more recently - SCUBA at the JCMT and the 19-channel array at the Heinrich-HertzTelescope have produced important results for the investigation of this cold dust component. Also, the ISO satellite could routinely measure the peak of the cold dust emission from grains with temperatures ranging down to $10 \mathrm{~K}$ (e.g. Popescu et al. 2002).

Send offprint requests to: M. Dumke, e-mail: mdumke@mpifr-bonn.mpg.de
However, dust properties in external galaxies are still not well determined because of gaps in the spectral coverage. Another difficulty in the analysis and interpretation of continuum data is the possible contribution of molecular lines to the total flux measured with the (broad-band) bolometer arrays, especially in the usually used atmospheric windows around $\lambda \lambda 870 \mu \mathrm{m}$ and $1.23 \mathrm{~mm}$ which contain the strong $\mathrm{CO}(3-2)$ and (2-1) lines. This situation currently improves as more and more objects are mapped even in the higher $\mathrm{CO}$ transitions.

This current paper is the first in a series that reports on observations of the thermal emission of cold dust in selected galaxies. NGC 4631 is a nearby $(D=7.5 \mathrm{Mpc}$, e.g. Golla \& Wielebinski 1994) galaxy which is close to edge-on. While the position angle of the large-scale (cm-) radio continuum emission is about $86^{\circ}$, that of the inner disk is closer to $82^{\circ}$. A value of $84^{\circ}$, which we assume throughout this paper, seems to be most appropriate for the $\mathrm{mm} / \mathrm{sub}-\mathrm{mm}$ emission out to a radius of a few arcminutes. NGC 4631 is embedded in a small gravitationally interacting group with two neighboring galaxies. The dwarf elliptical galaxy NGC 4627 is located only 3' northwest of the nucleus of NGC 4631, and another edge-on spiral, NGC 4656, is located about $30^{\prime}$ to the southeast. This interaction has created several prominent $\mathrm{H}$ I bridges and spurs (Rand 1994). Since galaxy interactions play an important role in the evolution of galaxy systems and the ISM content in galaxies, 
Table 1. Some basic parameters of NGC 4631 as obtained from the literature.

\begin{tabular}{lll}
\hline \hline $\begin{array}{l}\text { Type } \\
\text { Position: }\end{array}$ & Sd & $\begin{array}{l}\text { de Vaucouleurs et al. (1991) } \\
\text { Young et al. (1995) }\end{array}$ \\
RA[2000] & $12^{\mathrm{h}} 42^{\mathrm{m}} 07.65$ & \\
Dec[2000] & $32^{\circ} 32^{\prime} 27^{\prime} .9$ & \\
Distance & $7.5 \mathrm{Mpc}$ & Golla \& Wielebinski (1994) \\
Pos. Angle & $84^{\circ}$ & \\
Inclination & $86^{\circ}$ & \\
$M(\mathrm{H} \mathrm{I})$ & $5 \times 10^{9} M_{\odot}$ & Rand (1994) \\
$M\left(\mathrm{H}_{2}\right)$ & $1.2 \times 10^{9} M_{\odot}$ & Golla \& Wielebinski (1994) \\
IRAS flux densities: & Young et al. (1989) \\
$12 \mu \mathrm{m}$ & $5.1 \pm 1.1$ & \\
$25 \mu \mathrm{m}$ & $8.8 \pm 1.8$ & \\
$60 \mu \mathrm{m}$ & $90.7 \pm 18.2$ & \\
$100 \mu \mathrm{m}$ & $170.4 \pm 34.1$ & \\
\hline
\end{tabular}

the investigation of this object can provide useful information about many processes in the ISM.

NGC 4631, which was classified as "mild starburst" by Golla \& Wielebinski (1994), may be in a late stage of its interaction, where the central star formation (triggered by molecular inflow due to the gravitational forces) has already ceased and an energetic outflow as well as a huge radio halo have been formed (Ekers \& Sancisi 1977; Rand 2000).

The observations of the dust component of NGC 4631 started with the IRAS satellite (Rice et al. 1988; Young et al. 1989). While the IRAS observations were sensitive mainly to the warm dust present in this galaxy, the $\lambda 1.3 \mathrm{~mm}$ map of Braine et al. (1995) proved the existence of a significant amount of cold dust in the central area. The inner 2'.5 were also observed at $\lambda 850 \mu \mathrm{m}$ by Alton et al. (1999). Neininger $\&$ Dumke (1999) presented a more extended map at $\lambda 1.23 \mathrm{~mm}$ (which was obtained from the same data as shown in this paper) and detected intergalactic cold dust which was pulled out of the disk by the gravitational interaction of NGC 4631 with its neighbours. Here we present a more extended map at $\lambda 870 \mu \mathrm{m}$, covering the disk out to a radius of $7^{\prime}$.

Some basic parameters of NGC 4631 are compiled in Table 1.

\section{Observations and data reduction}

We observed the galaxy NGC4631 at wavelengths of $\lambda \lambda 870 \mu \mathrm{m}$ and $1.23 \mathrm{~mm}$, using sensitive 19-channel bolometer arrays developed by E. Kreysa and collaborators at the MaxPlanck-Institut für Radioastronomie, Bonn.

\subsection{HHT observations}

The observations at $\lambda 870 \mu \mathrm{m}$ were carried out at the HeinrichHertz-Telescope $^{1}$ (Baars et al. 1999), located on Mt. Graham, Arizona, during three observing sessions between March 2000 and January 2002, using a 19-channel bolometer array installed as facility instrument. The 19 channels of this bolometer are

\footnotetext{
1 The HHT is operated by the Submillimeter Telescope Observatory on behalf of Steward Observatory and the MPI für Radioastronomie.
}

located in the centre and on the sides of two concentric regular hexagons, with an apparent spacing between two adjacent channels (beams) of 50". The central frequency of the bolometer is about $345 \mathrm{GHz}$ (the highest sensitivity is reached at $340 \mathrm{GHz}$ ), and the instrument is sensitive mainly between 310 and $380 \mathrm{GHz}$.

In order to calculate the atmospheric zenith opacity, we made skydip observations every 45 to $90 \mathrm{~min}$, depending on the atmospheric stability. These yielded atmospheric opacities at our observing frequency which varied between 0.3 and 0.9 for the several observing sessions. For calibration purposes we have also performed mapping and on-off measurements of various planets (mainly Mars and Saturn) during the observations. These measurements yielded a conversion factor from observed counts to $\mathrm{mJy} / \mathrm{beam}$ of $0.8-1.1 \mathrm{mJy}^{\mathrm{beam}}{ }^{-1}$ count $^{-1}$. This varying conversion factor is due to (and partly corrects for) varying atmospheric condition at the day of the observations and the uncertainties in the opacity calculation. The beamwidth at this frequency is $\sim 23^{\prime \prime}$.

All maps were observed in the Az-El coordinate system, by scanning along Azimuth and with data acquisition every $0.5 \mathrm{~s}$. During the observations, the subreflector was wobbled at $2 \mathrm{~Hz}$ in azimuth, with a beam throw between $60^{\prime \prime}$ and $200^{\prime \prime}$. If necessary, the starting point of each subscan was shifted by a few arcseconds with respect to the preceding one, in order to ideally place each individual coverage relative to the target source. This as well as different map sizes (typically around $\left.450^{\prime \prime} \times 300^{\prime \prime}\right)$ ensured that each subscan covers the galaxy and a sufficient amount of blank sky on either side, in order to facilitate baseline subtraction and a correct restoration of the doublebeam maps.

\subsection{Pico Veleta observations}

The observations at $\lambda 1.23 \mathrm{~mm}$ were carried out in March 1997 at the IRAM 30-m telescope on Pico Veleta, Spain. The central frequency of the used bolometer array is $243 \mathrm{GHz}$, the bandwidth about $70 \mathrm{GHz}$. The array layout is similar to the one at the HHT, with individual channels separated by $20^{\prime \prime}$. The beamwidth at the observing frequency is $11^{\prime \prime}$.

The sky opacity at the observing frequency was monitored with skydips and varied between 0.1 and 0.3 during the five days of the observing run, but was stable within \pm 0.02 for each individual day of the observations. We mapped Mars every night to determine the absolute flux density scale; from these measurements we obtained a conversion factor of 0.24 0.28 mJy beam $^{-1}$ count $^{-1}$.

The observing procedures were similar to the HHT observations. The maps were observed in the Az-El coordinate system, but with a lower scanning velocity and smaller subscan separation in order to ensure full sampling. The subreflector was wobbled at $2 \mathrm{~Hz}$ with a beam throw of $45^{\prime \prime}$.

\subsection{Data reduction}

The data reduction for the HHT and the 30-m data was performed with the NIC program of the GILDAS software 


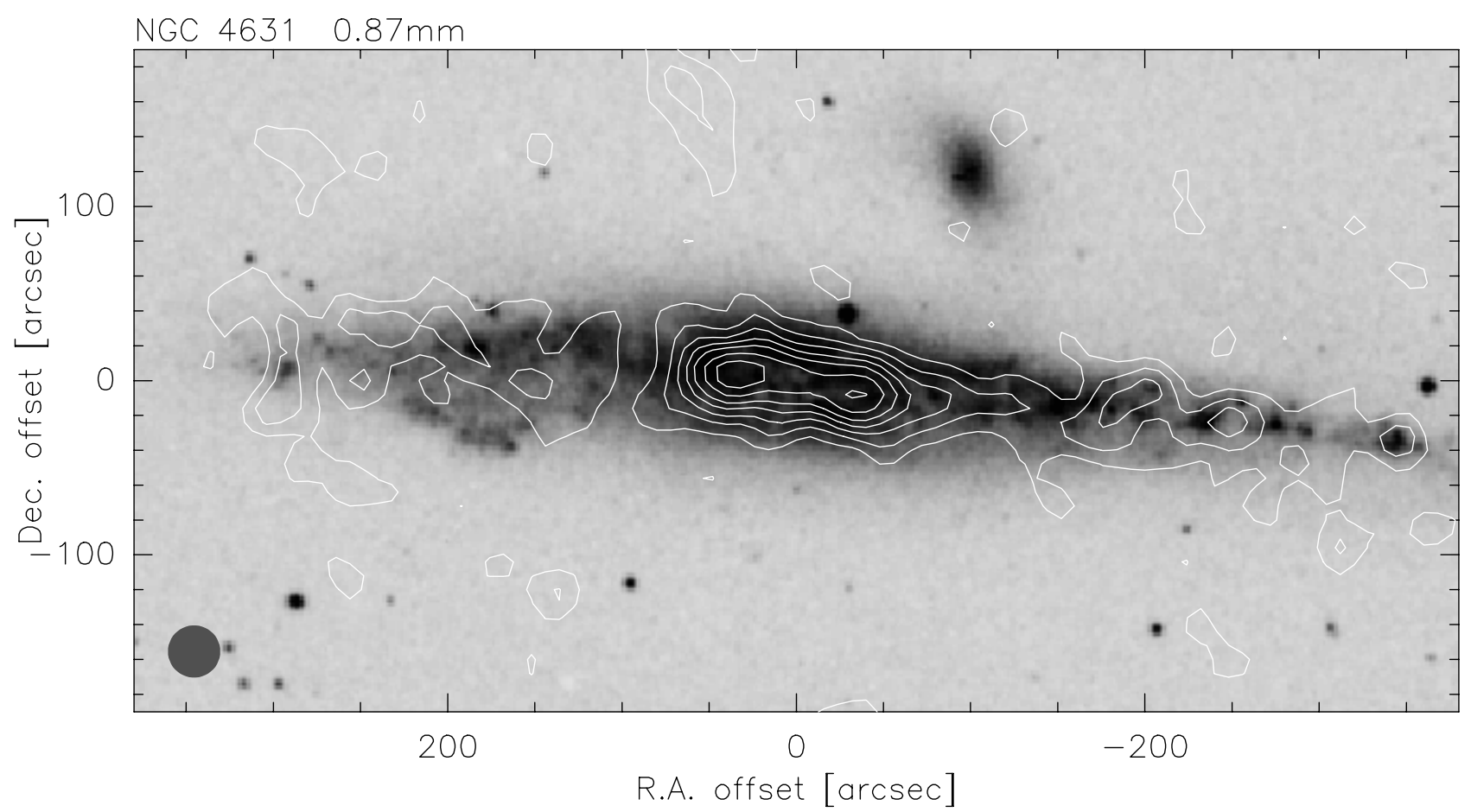

Fig. 1. Continuum map of NGC 4631 at $\lambda 0.87 \mathrm{~mm}$, overlaid on an optical image extracted from the Digitized Sky Survey. The map is smoothed to an angular resolution of $30^{\prime \prime}$; the beam size is indicated by the filled grey circle in the lower left corner. The rms noise in the map is about $20 \mathrm{mJy} / \mathrm{beam}$ area, and contour levels are 40,80,120,160,200,250,300 mJy/beam area. The dark spot in the optical (greyscale) image $3^{\prime}$ northwest of the central area is the neighbouring galaxy NGC 4627.

package. In addition, we used the MOPSI program to confirm (and adjust, if necessary) the zenith opacities calculated by NIC from our skydip measurements, and also to estimate flux densities for the observed planets in order to calibrate the data in astronomical units. After baseline subtraction and the elimination of spikes in each single coverage, the atmospheric noise, which is highly correlated between the individual channels (especially at $870 \mu \mathrm{m}$ ), was subtracted. The maps were gridded, restored (using the EKH algorithm), converted into the RA-Dec system and finally combined (with an appropriate weighting) to a single map for each of the two wavelengths. The zero-levels of the resulting maps were checked and carefully adjusted. Map features smaller than the telescope beam appearing in the final maps were filtered out using a Fourier filter technique. For further analysis, both maps were smoothed to a FWHM of $24^{\prime \prime}$. This was done in order to enable a direct comparison with the existing CO data (see Sect. 3.3) and other ISM components. The rms noise level in the final maps is $20 \mathrm{mJy} /$ beam at $\lambda 0.87 \mathrm{~mm}$, and $3 \mathrm{mJy} /$ beam at $\lambda 1.23 \mathrm{~mm}$.

\section{Results}

\subsection{Total flux densities}

To measure the total flux densities, we used a ring integration method which we applied to the disk of NGC 4631. While the parameter choice for this ring integration had some influence on the results, its effect was much smaller than the uncertainties coming from the observations and the data reduction process, including the calibration. Also the actual rms noise in the final maps has a rather small effect. We estimate the error of the flux densities to be $10 \%$ at $\lambda 1.23 \mathrm{~mm}$, and $15 \%$ at $\lambda 0.87 \mathrm{~mm}$.

The total flux density of NGC 4631, including all disk emission, is $S_{0.87 \mathrm{~mm}}=3.78 \pm 0.57 \mathrm{Jy}$ for the observing wavelengths of $0.87 \mathrm{~mm}$. At $\lambda 1.23 \mathrm{~mm}$, the total flux density is more difficult to determine. The reason for this are the bridges and spurs which were detected at this wavelength, as well as the fact that the edge of the detected emission is more due to the edge of the mapped region rather than due to the edge of the dust distribution. Limiting the integration of the observed emission to the main disk of NGC 4631, we estimate $S_{1.23 \mathrm{~mm}}=2.18 \pm 0.22 \mathrm{Jy}$. Note that we got these values from integrating over the same area and using maps at the same angular resolution (24") for both wavelengths.

These values cannot be easily compared to previous flux density estimates, since our maps are the first to cover (almost) all of the galaxy's emission. Braine et al. (1995) observed the central $4^{\prime} \times 3^{\prime}$ of NGC 4631 at a wavelength of $1.3 \mathrm{~mm}$ and obtained a flux density of $0.64 \mathrm{Jy}$. When we restrict our integration to the same area, we measure $\sim 0.9 \mathrm{Jy}$. This difference is only partly due to the slightly different wavelength. Another reason for their lower value might be the lack of sensitivity for the off-plane emission and therefore an imperfect baseline subtraction - note the negative areas north and south of the major axis and the overall smaller intensity values in Fig. 1 of Braine et al. (1995).

Bendo et al. (2003) used SCUBA archive data to estimate the flux density at $\lambda 850 \mu \mathrm{m}$ and obtained $1.89 \pm 0.19 \mathrm{Jy}$ and $0.54 \pm 0.05 \mathrm{Jy}$ for the central $135^{\prime \prime}$ and $45^{\prime \prime}$, respectively. 


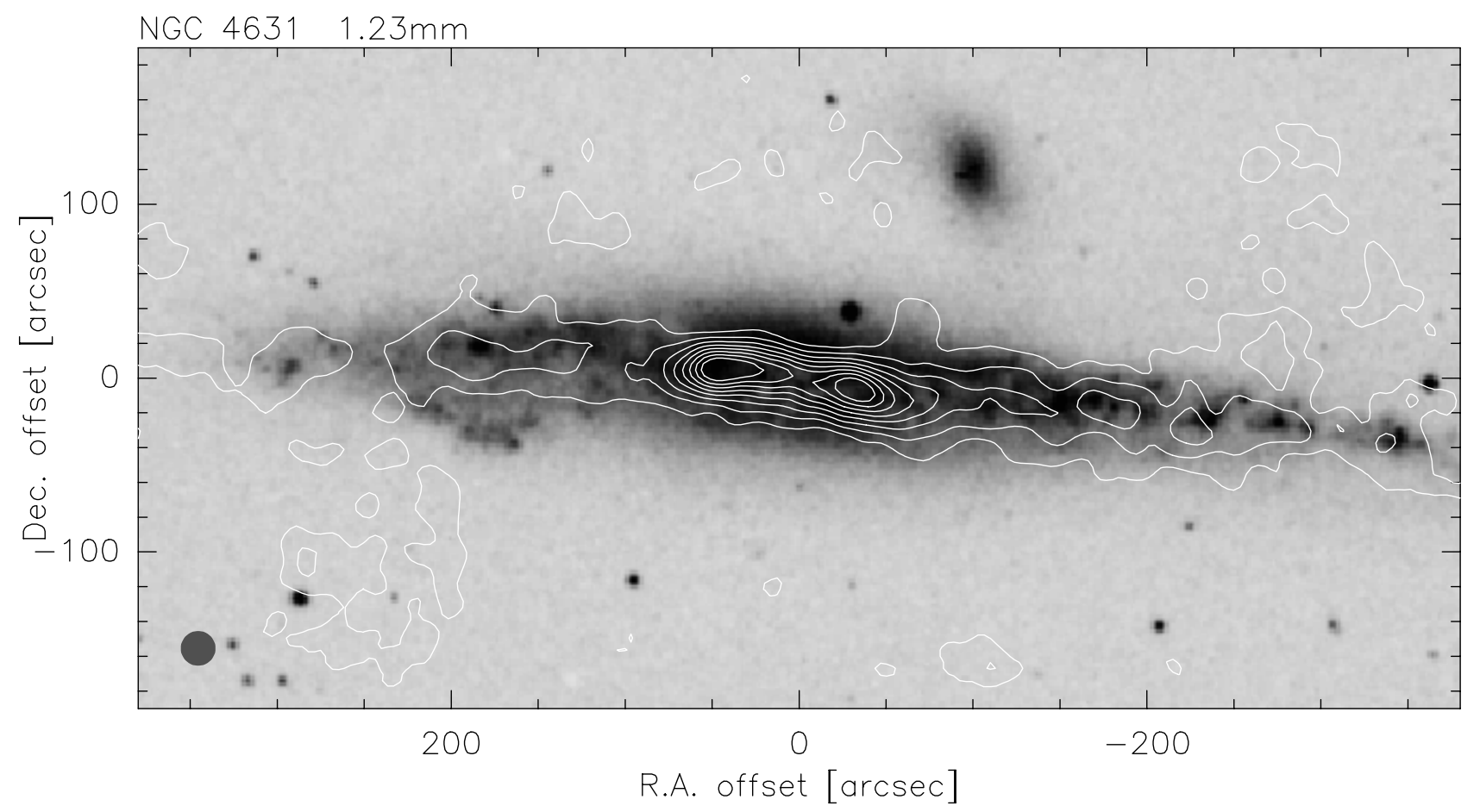

Fig. 2. Continuum map of NGC 4631 at $\lambda 1.23 \mathrm{~mm}$, overlaid on an optical image extracted from the Digitized Sky Survey. The map is smoothed to an angular resolution of $20^{\prime \prime}$; the beam size is indicated by the filled grey circle in the lower left corner. The rms noise in the map is about $3 \mathrm{mJy} /$ beam area, and contour levels are $10,20, \ldots, 80 \mathrm{mJy} /$ beam area.

For the same areas, we get values of $1.8 \mathrm{Jy}$ and $0.5 \mathrm{Jy}$, which is in good agreement with those values.

\subsection{Morphology of the continuum maps}

The resulting maps of NGC 4631 at $\lambda \lambda 0.87 \mathrm{~mm}$ and $1.23 \mathrm{~mm}$ are shown in Figs. 1 and 2, overlaid on an optical image extracted from the Digitized Sky Survey.

Similar to other ISM components, especially the CO molecular line emission, both maps show a double-peaked appearance in the central area, which points to a ring-like distribution with a diameter of about $1^{\prime}$, or $2.2 \mathrm{kpc}$ at the assumed distance of $7.5 \mathrm{Mpc}$.

At larger radii, the emission is more patchy and follows the major axis of the galaxy. The extent of the emission in the disk is difficult to determine, since our map does not cover radii larger than $6^{\prime}-7^{\prime}$ with a sufficient signal-to-noise ratio. At $\lambda 1.23 \mathrm{~mm}$ we find a significant amount of emission at distances of a few arcminutes away from the major axis, e.g. at $(\Delta \alpha, \Delta \delta)=\left(250^{\prime \prime},-150^{\prime \prime}\right)$ or $\left(0^{\prime \prime}, 120^{\prime \prime}\right)$, and thus several $\mathrm{kpc}$ above the plane. This emission is correlated with the H I spurs found by Rand (1994) and is discussed in detail by Neininger \& Dumke (1999). It is most likely interstellar material pulled out of the plane by the gravitational interaction of NGC 4631 with its neighbouring galaxies. The total flux density of this extraplanar gas - as far as it is within our map edges - is about $0.5 \mathrm{Jy}$. This is one quarter of the flux density within the plane of NGC 4631, which is $2.18 \mathrm{mJy}$.

At $\lambda 0.87 \mathrm{~mm}$ we are unable to detect this intergalactic cold dust with sufficient significance. Only one area, located at $(\Delta \alpha, \Delta \delta)=\left(60^{\prime \prime}, 170^{\prime \prime}\right)$, is clearly detected. This feature is located on the H I spur pointing towards the northeast as seen by e.g. Weliachew et al. (1978). None of the other extraplanar features of the $1.23 \mathrm{~mm}$ map can be seen above the noise in the sub-mm map. However, an integration of the map shows that a flux density of $\sim 1$ Jy originates in the off-plane features which can be identified in the $1.23 \mathrm{~mm}$ map, of which $400 \mathrm{mJy}$ are due to the emission region described above, located northeast of the nucleus. The total flux density confined to the plane of NGC 4631 and located within our map is $3.78 \pm 0.57 \mathrm{Jy}$, so again the off-plane features contain about $25 \%$ of the disk emission.

The radiation measured by the bolometers contain some contributions which are not due to thermal dust emission. We calculate these non-dust contributions in the next subsection, before we continue with the discussion of the major axis distribution of important ISM components and the dust spectrum.

\subsection{Origin of the observed emission}

The broad band emission of the galaxies, measured with the bolometers, consists of four main components: thermal dust emission, free-free radiation from thermal electrons, synchrotron radiation from relativistic electrons, and the $\mathrm{CO}$ as well as some weaker lines which fall into the bandpass. As we are mainly interested in the thermal dust emission alone, we have to determine the contribution of the other processes and to subtract them from the data. 
Since CO mapping observations of NGC 4631 exist, we can calculate $B_{\mathrm{CO}}$, the contribution of the $\mathrm{CO}$ lines to the surface brightness measured with the bolometers, through

$B_{\mathrm{CO}}=\frac{2 k v^{3} c^{-3}}{\Delta v_{\text {bol }}} \Omega_{\text {beam }} I_{\mathrm{CO}}$,

where the line intensity $I_{\mathrm{CO}}=\int T_{\mathrm{mb}} \mathrm{d} v$ denotes the velocityintegrated main-beam brightness temperatures. From the integrated $\mathrm{CO}$ line flux $F_{\mathrm{CO}}$, the contribution to the total flux densities can be obtained via

$S_{\mathrm{CO}}=\frac{v c^{-1}}{\Delta v_{\mathrm{bol}}} F_{\mathrm{CO}}$.

Here the equivalent bandwidth of the bolometer can be calculated from the instrumental bandpass (Kreysa, priv. comm.) and is $\Delta v_{\mathrm{bol}} \sim 50 \mathrm{GHz}$ for the $\mathrm{CO}(3-2)$ line and $\Delta v_{\mathrm{bol}} \sim 70 \mathrm{GHz}$ for the $\mathrm{CO}(2-1)$ line.

The contribution of the $\mathrm{CO}(3-2)$ to the $870 \mu \mathrm{m}$ flux can be taken from data published by Dumke et al. (2001), and the $\mathrm{CO}(2-1)$ contribution to the $1.23 \mathrm{~mm}$ flux can be estimated from the data published by Golla \& Wielebinski (1994). We should note that at both wavelengths the $\mathrm{CO}$ emission was observed with the same telescope as the corresponding continuum emission, thus eliminating possible uncertainties due to beam characteristics. The total line contribution, including also ${ }^{13} \mathrm{CO}$ and other lines, may be somewhat higher. Based on typical line ratios in external galaxies and the sensitivity variation of the bolometer over the bandpass, we estimate that other lines add up to about $5 \%$ of the flux calculated from the ${ }^{12} \mathrm{CO}(3-2)$ line, and about $10 \%$ of the flux from the ${ }^{12} \mathrm{CO}(2-1)$ line. Taking this into account and entering all quantities in Eq. (1), it simplifies to

$B_{\text {line }}^{0.87 \mathrm{~mm}}[\mathrm{mJy}]=1.36 I_{\mathrm{CO}(3-2)}\left[\mathrm{K} \mathrm{km} \mathrm{s}^{-1}\right]$

and

$B_{\text {line }}^{1.23 \mathrm{~mm}}[\mathrm{mJy}]=0.303 I_{\mathrm{CO}(2-1)}\left[\mathrm{K} \mathrm{km} \mathrm{s}^{-1}\right]$

for the $\lambda \lambda 870 \mu \mathrm{m}$ and $1.23 \mathrm{~mm}$ emission, respectively, and a $24^{\prime \prime}$ beam. To correct for the line contribution in the continuum maps, we scaled the $\mathrm{CO}$ intensity maps accordingly and subtracted these from the bolometer maps. For a comparison of the dust distribution at both wavelengths with other ISM components (see next subsection) we smoothed all maps to a final resolution of 24" HPBW.

For the line contributions to the total flux denity, we get $S_{\text {line }}^{0.87 \mathrm{~mm}}=330 \pm 30 \mathrm{mJy}$ and $S_{\text {line }}^{1.23 \mathrm{~mm}}=90 \pm 10 \mathrm{mJy}$. The fraction of this contribution to the observed flux density is thus $9 \%$ and $4 \%$ for the two wavelengths of $0.87 \mathrm{~mm}$ and $1.23 \mathrm{~mm}$, respectively.

The fraction of the free-free and the synchrotron emission can be estimated from radio continuum data at lower frequencies. Niklas et al. $(1995,1997)$ separated these two components in the radio spectra of a large sample of galaxies. At a frequency of $10 \mathrm{GHz}$ they found a thermal fraction of the emission of $f_{\text {th }}=0.13$ and a nonthermal spectral index of $\alpha_{\text {nth }}=0.78$. With these values, we estimate a contribution of the free-free and synchrotron emission at both wavelengths of $40 \pm 10 \mathrm{mJy}$
Table 2. Flux densities of NGC 4631 at $\lambda \lambda 0.87 \mathrm{~mm}$ and $1.23 \mathrm{~mm}$. The non-dust contributions at both wavelengths are calculated as described in Sect. 3.3.

\begin{tabular}{ccccc}
\hline \hline$\lambda$ & $S_{\text {obs }}[\mathrm{Jy}]$ & $S_{\text {line }}[\mathrm{mJy}]$ & $S_{\mathrm{ff}+\text { sync }}[\mathrm{mJy}]$ & $S_{\mathrm{dust}}[\mathrm{Jy}]$ \\
\hline $0.87 \mathrm{~mm}$ & $3.78 \pm 0.57$ & $330 \pm 30$ & $40 \pm 10$ & $3.41 \pm 0.58$ \\
$1.23 \mathrm{~mm}$ & $2.18 \pm 0.22$ & $90 \pm 10$ & $50 \pm 10$ & $2.04 \pm 0.23$ \\
\hline
\end{tabular}

$(0.87 \mathrm{~mm})$ and $50 \pm 10 \mathrm{mJy}(1.23 \mathrm{~mm})$, which is of the order of $1-2 \%$ of the total emission. Although the thermal fraction of the $\mathrm{cm}$-emission might be underestimated because of galactic wind and cosmic ray propagation effects on the integrated radio spectrum (Werner 1988), the contribution of free-free and synchrotron radiation is still less than $80 \mathrm{mJy}$ at both wavelengths even in the unlikely case that more than half of the emission in the $\mathrm{cm}$-range is free-free emission. Therefore it is much smaller than the line contribution, especially at $\lambda 0.87 \mathrm{~mm}$. Thus any uncertainties in the assumed thermal (free-free) fraction of the $\mathrm{cm}$-emission and the non-thermal spectral index are negligible for the further data analysis, considering the total uncertainty of the flux density values of $10-15 \%$.

The contributions of the line emission and the non-dust continuum radiation to the total flux densities at $0.87 \mathrm{~mm}$ and $1.23 \mathrm{~mm}$ are listed in Table 2. At this point we should note that the main uncertainties in the results are due to the rms noise in and the absolute calibration of the continuum maps. The subtraction (or non-subtraction) of the non-dust contribution does not change our results qualitatively, and the uncertainties in these contributions are negligible.

\subsection{ISM distribution along the major axis}

We determined the distribution of various components of the ISM along the major axis of NGC 4631, including the current results from $\lambda \lambda 0.87$ and $1.23 \mathrm{~mm}$ (after subtraction of the nondust contributions), the H I (Rand 1994), the CO(1-0) (Golla \& Wielebinski 1994), and the $\lambda 6 \mathrm{~cm}$ radio continuum emission (Krause et al., in prep.). The result is shown in Fig. 3, with an angular resolution of $24^{\prime \prime}$ for all data sets. Here we averaged the data over three pixels (pixel size is $8^{\prime \prime}$ ) perpendicular to the major axis for each data point in order to increase the signal-tonoise ratio, and to account for emission which is close to, but not exactly on the major axis. The $\lambda 6 \mathrm{~cm}$ data are included as a tracer for the FIR emission, since at this wavelength we see mainly non-thermal radiation, for which the radio-FIR correlation is strongest.

Similar to other edge-on galaxies, the dust emission resembles the distribution of molecular gas in the inner part of the galaxy. However, at radii where no molecular gas can be detected $\left(r>4^{\prime}\right)$, there is still a significant amount of thermal dust emission, which in this case follows the distribution of the H I.

By comparing the distributions for the various wavelengths of radio continuum emission, we note some significant differences between the data sets. The central maximum is only visible in the $\lambda 6 \mathrm{~cm}$ data, but not at $\mathrm{mm} / \mathrm{sub}-\mathrm{mm}$ wavelengths. Since the existence of an active nucleus, capable to produce 


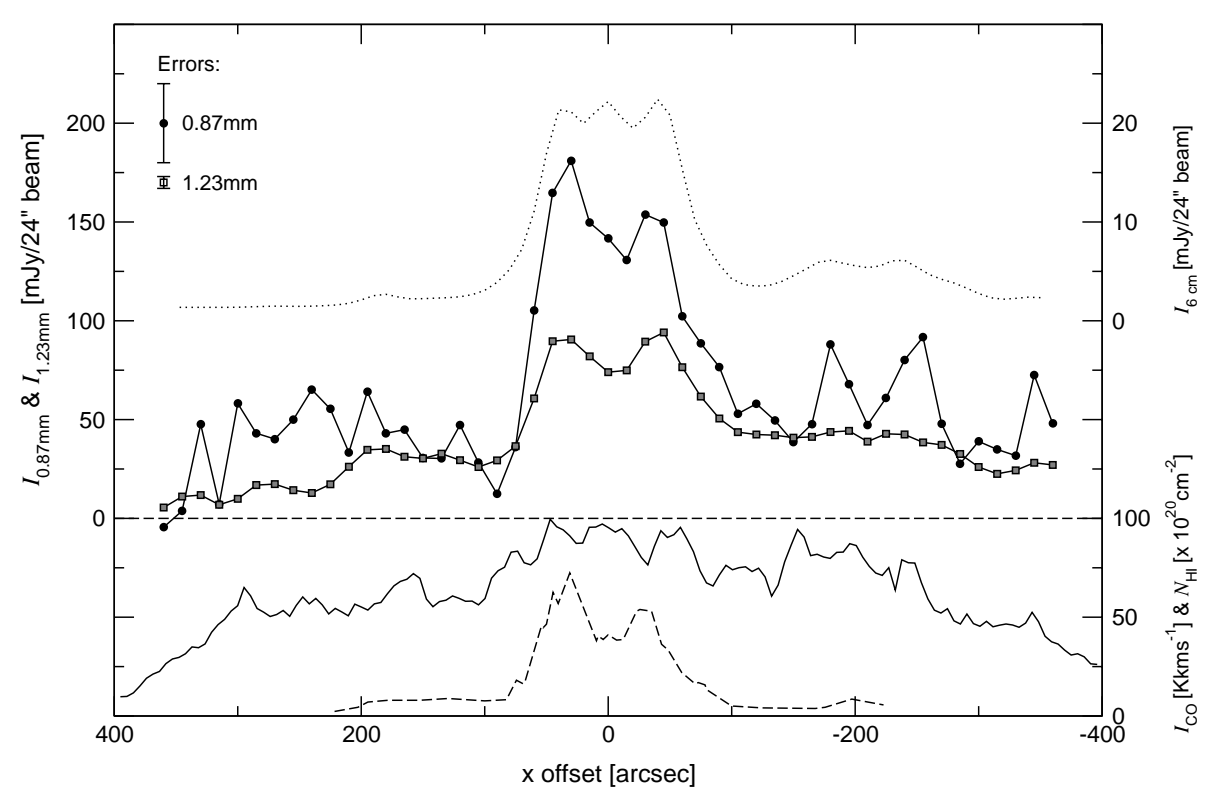

Fig. 3. Distribution of various ISM components along the major axis of NGC 4631. The thermal dust emission at $870 \mu \mathrm{m}$ and $1.23 \mathrm{~mm}$, as obtained from the maps in Figs. 1 and 2 and corrected for line, synchrotron and free-free contributions, are shown as filled circles and white squares. The errors of both data sets are indicated in the upper left corner, while the longdashed line gives their zero level. Also shown for comparison are the HI profile (solid line), the $\mathrm{CO}(1-0)$ distribution (dashed line), and the $\lambda 6 \mathrm{~cm}$ radio continuum distribution (dotted line). The scale for these three data sets is shown at the $y$-axis on the right. excess cm emission, is rather unlikely in NGC 4631, this points to a steeper FIR-to-mm spectrum and thus to higher dust temperatures in the very centre. Also the intensity of the "dust ring" is different at the eastern and western maximum, suggesting different dust properties in these two maxima. All distributions show a steep decrease in intensity at the eastern edge of the molecular ring, at a radius of $40^{\prime \prime}-70^{\prime \prime}$, while the intensity decrease at the western edge is much shallower.

At the molecular ring itself, at a radius of about $30^{\prime \prime}$, all continuum distributions show local maxima. Such a correlation between molecular gas and dust is expected because many molecules are formed on the surface of dust grains, and the dust shields the gas from starlight and prevents the molecules from being photodissociated. However, the $\mathrm{H}$ I distribution shows local minima at these positions. Here the interstellar hydrogen was probably transformed into molecules because of a local increase of ambient density. This is a direct consequence of gas flows due to a non-axisymmetric potential, which results from the gravitational interaction with the neighbouring galaxies NGC 4656 and NGC 4627.

While the CO intensities have dropped to relatively low values beyond the molecular ring, the H I emission stays at significant levels. Also the tracers of interstellar dust $(\lambda \lambda 1.23 \mathrm{~mm}$, $0.87 \mathrm{~mm}$, and $6 \mathrm{~cm}$, i.e. FIR) are still detected at larger radii. However, different correlations between the various ISM components can be observed. In the western disk, $3^{\prime}-4^{\prime}$ away from the centre, the H I distribution shows a plateau-like feature. Here also the $0.87 \mathrm{~mm}$ and $6 \mathrm{~cm}$ data show maxima in their distribution, while we see only a small increase in the $1.23 \mathrm{~mm}$ data. In the eastern disk, on the other hand, the $1.23 \mathrm{~mm}$ and $6 \mathrm{~cm}$ distributions drop beyond a radius of $3^{\prime}$, while the $0.87 \mathrm{~mm}$ emission stays at a higher level, similar to the H I. These significant differences in the distribution of the various dust tracers point to changing dust properties along the major axis of NGC 4631. This will be discussed in more detail in Sect. 4.3.

\section{Dust properties in NGC 4631}

\subsection{The observed dust spectrum}

Table 2 lists the observed flux densities for both wavelengths. In addition, it gives the non-dust contributions to the flux densities and the resulting values which are due to the thermal emission of dust alone. As discussed in Sect. 3.3, the line contribution of the measured continuum flux densities is $9 \%$ at $\lambda 0.87 \mathrm{~mm}$, and $4 \%$ at $\lambda 1.23 \mathrm{~mm}$. The latter value is significantly below this value measured in other galaxies, either normal (e.g. NGC 5907, Dumke et al. 1997), active (e.g. NGC 3079, Braine et al. 1997), or starbursting (e.g. M 82, Thuma et al. 2000). There the line contribution to the measured continuum flux ranges from $8 \%$ in NGC 5907 to $35 \%$ in the centre of NGC 3079, and it seems to be correlated with the activity status of the galaxy. This would point to NGC 4631 being a rather inactive galaxy. However, a small fraction of line emission can also point to a higher value for the dust emission per gas mass, i.e. to a higher absorption cross section (see further below).

The two measured flux density values at $\lambda \lambda 0.87$ and $1.23 \mathrm{~mm}$, together with the IRAS data (Young et al. 1989), still leave a large gap in the spectral coverage between the FIR and the sub-mm. In order to fill this gap, we take the ISO and SCUBA data published by Bendo et al. $(2002,2003)$ and estimate flux density values for wavelengths of $180 \mu \mathrm{m}$ and $450 \mu \mathrm{m}$.

Concerning the SCUBA data, these authors give a flux density value only for the inner $135^{\prime \prime}$ of the galaxy. In order to estimate the total flux density for NGC 4631, we have to scale this by a factor $S_{\text {tot }} / S_{135^{\prime \prime}}$, describing the ratio of the total flux density to the flux density in the central $135^{\prime \prime}$ of the galaxy. From our maps, we measured this ratio to 2.27 and 2.34 at a wavelength of $0.87 \mathrm{~mm}$ and $1.23 \mathrm{~mm}$, respectively (after subtraction of the non-dust contributions, which are negligible at $\lambda 450 \mu \mathrm{m})$. In the FIR, the ratio of IRAS flux densities (corresponding to the total emission) to the ISO values (corresponding to the inner $135^{\prime \prime}$ ) is about 1.6 at $\lambda 60 \mu \mathrm{m}$ and 1.5 
at $\lambda 100 \mu \mathrm{m}$, where the latter value is less certain due to the wider PSF of ISOPHOT at this wavelength. Considering that the warmer dust (i.e. at shorter wavelengths) is probably more concentrated to the inner disk than the colder dust, we assume a factor of $S_{\text {tot }} / S_{135^{\prime \prime}}=2.0$ at $\lambda 450 \mu \mathrm{m}$. With this value we get a total flux density of $S_{450 \mu \mathrm{m}}=36 \pm 9 \mathrm{Jy}$.

In order to estimate the total flux density at $\lambda 180 \mu \mathrm{m}$, we take into account that the ISOPHOT C 200 detector array covers only the central $180^{\prime \prime} \times 180^{\prime \prime}$ of the galaxy, and therefore also misses a significant fraction of the disk emission. Again we can estimate a scaling factor from our maps, and find $S_{\text {tot }} / S_{180^{\prime \prime}} \sim 1.8$ for $\lambda 180 \mu \mathrm{m}$. Interestingly, we find at $\lambda \lambda 0.87 \mathrm{~mm}$ and $1.23 \mathrm{~mm}$ that the flux density within the central $180^{\prime \prime}$ is not much larger than that within the central $135^{\prime \prime}$. This can be understood by the fact that the smaller $135^{\prime \prime}$ area covers already the central "dust ring", as described in the previous section. This result differs from the values given by Bendo et al. (2003), who found a much larger difference between the two flux density values for the inner $135^{\prime \prime}$ and the inner $180^{\prime \prime}$. This inconsistency can probably be explained by the fact that these authors used a SCUBA sub-mm map for a deconvolution analysis, although this map covers less then $3^{\prime}$ of the inner disk, and is therefore not well suited for this purpose.

In addition, the spatial resolution of ISO at this wavelength is not much smaller than the detector array. With the knowledge of the PSF of this detector one can determine which fraction of the total emission of a source would be detected by the C 200 . For a somewhat extended source like NGC 4631 we estimate a value of $82 \%$. Taking these two corrections into account, we get a total flux density at this wavelength of $S_{180 \mu \mathrm{m}}=205 \pm 35 \mathrm{Jy}$.

\subsection{Dust temperatures and composition}

The interstellar dust in galaxies is a mixture of many components at several temperatures. Although the exact composition and temperature distribution depends on the local interstellar radiation field and local processes important for dust grain processing, the total FIR to mm-spectrum of a galaxy can usually be fitted well by 1-3 components with a modified black-body spectrum of the form

$S_{\lambda} \propto \sigma_{\lambda}^{\mathrm{H}} B_{\lambda}\left(T_{\mathrm{d}}\right)$,

with the dust absorption cross section per hydrogen atom $\sigma_{\lambda}^{\mathrm{H}} \propto$ $\lambda^{-\beta}$. The value of $\beta$ depends on the dust composition and is still a matter of debate; theoretical arguments suggest values between 1 and 2. Realistic dust models, which are able to explain the observed dust emission and extinction, suggest $\beta \sim 2$ for big grains which radiate in thermal equilibrium with the ambient temperature (Andriesse 1974; Draine \& Lee 1984). In fact the FIR-to-mm spectra of many external galaxies have been successfully fitted with one or two components of dust with $\beta=2$ (e.g. Chini et al. 1995; Neininger et al. 1996; Dumke et al. 1997; Braine et al. 1997; Alton et al. 1998; Stevens \& Gear 2000).

We fitted the FIR-to-mm spectrum of NGC 4631 with a dust model consisting of two components, both with $\beta=2$. We used data points from Young et al. (1989) in the FIR $(25-100 \mu \mathrm{m})$,

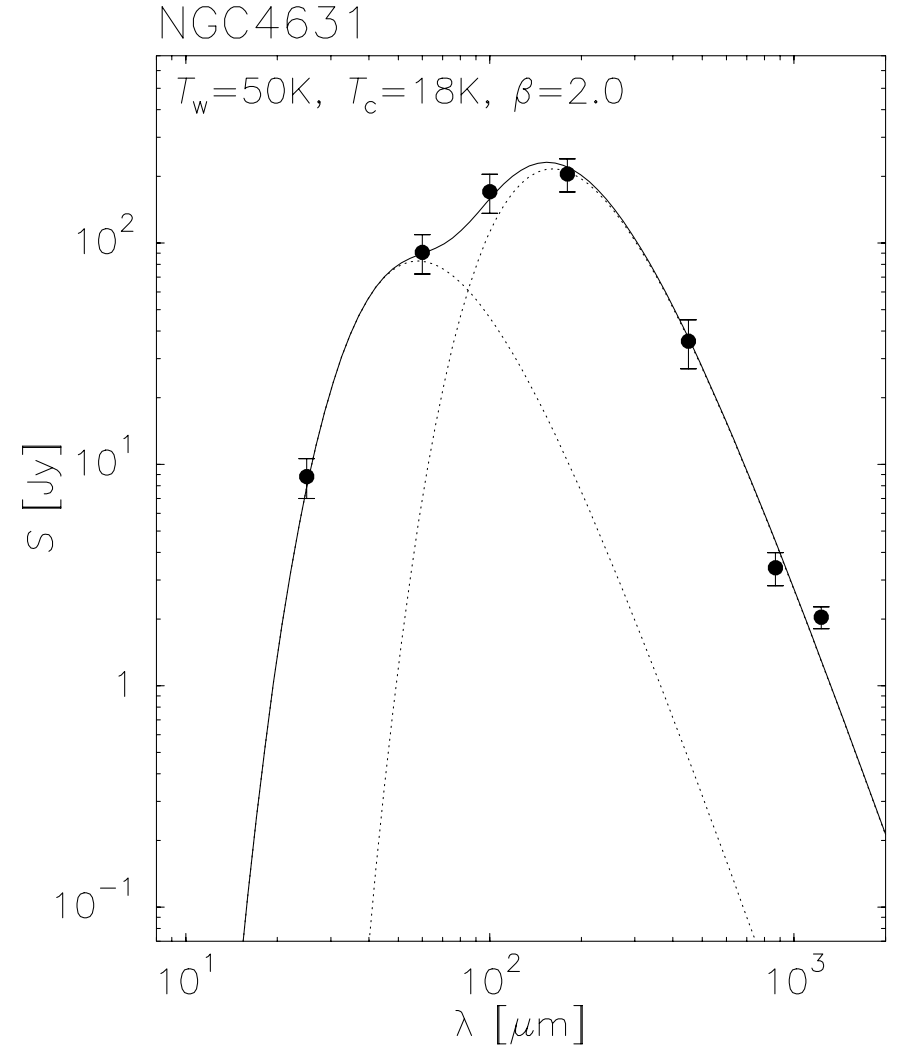

Fig. 4. The FIR-to-mm spectrum of NGC 4631. The FIR data points up to $\lambda 100 \mu \mathrm{m}$ are taken from Young et al. (1989), those at $180 \mu \mathrm{m}$ and $450 \mu \mathrm{m}$ are estimated from the values in Bendo et al. (2003), and the $0.87 \mathrm{~mm}$ and $1.23 \mathrm{~mm}$ flux densities are from this paper and corrected for line contributions and synchrotron and free-free emission. The solid line shows a fit for a "standard" dust model (two modified Planck curves with $\beta=2$ ).

those estimated above at $180 \mu \mathrm{m}$ and $450 \mu \mathrm{m}$ from Bendo et al. (2003), and our new values at $0.87 \mathrm{~mm}$ and $1.23 \mathrm{~mm}$. The result is shown in Fig. 4, where the solid curve represents a two-component modified Planck spectrum with temperatures of $50 \mathrm{~K}$ and $18 \mathrm{~K}$. This temperatures agree well with previous results (see references above), where the cold dust component was usually found to have a temperature of 15-20 K.

We can also estimate dust temperatures for the inner and outer disk separately when we use the flux density values at $\lambda \lambda 60 \mu \mathrm{m}$ and $100 \mu \mathrm{m}$ from Bendo et al. (2003) for the inner $135^{\prime \prime}$, and restrict the integration in our maps to the same area. A fit to the data leads to somewhat higher dust temperatures of $51 \mathrm{~K}$ and $20 \mathrm{~K}$ for the warm and cold component, respectively. After subtracting the flux density values for the inner disk from the total flux densities, we also estimate dust temperatures for the outer disk and find $49 \mathrm{~K}$ for the warm component and $16 \mathrm{~K}$ for the cold component. Please note, however, that this two-component model is still a model, and the main conclusion from these fits is that the dust temperature decreases from the centre of NGC 4631 to the outer disk.

As it can be seen in Fig. 4, this standard model seems to be not sufficient to explain the measured flux densities at both 0.87 and $1.23 \mathrm{~mm}$. Instead we detect an excess in the mm-range (or, alternatively, a lack of emission in the sub-mm): the ratio of 
the two dust-only flux densities (i.e. after subtraction of line and other contributions) is $S_{\text {dust }}(0.87 \mathrm{~mm}) / S_{\text {dust }}(1.23 \mathrm{~mm})=$ $1.67 \pm 0.35$, while we would expect a ratio of 4 in case of a modified Planck spectrum with a dust absorption coefficient $\propto \lambda^{-2}$. This excess of dust emission at $1.23 \mathrm{~mm}$ leads to a flattening of the sub-mm/mm spectrum of NGC 4631, which is difficult to explain with the thermal radiation of big dust grains at moderately warm temperatures alone. However, for practical reasons, we continue here with the analysis of the data by assuming that the above model is reasonable, and discuss this mm excess further below.

\subsection{Absorption cross sections}

An averaged value of the dust absorption cross section in NGC 4631 can be calculated from the measured flux density and the hydrogen mass through (e.g. Hildebrand 1983)

$\sigma_{\lambda}^{\mathrm{H}}=\frac{S_{\lambda} m_{\mathrm{H}} D^{2}}{B_{\lambda}\left(T_{\mathrm{d}}\right) M_{\mathrm{H}}}$.

Here $m_{\mathrm{H}}$ is the mass of a hydrogen atom, $D$ the distance to the galaxy, and $M_{\mathrm{H}}=M_{\mathrm{HI}}+M_{\mathrm{H}_{2}}$ the hydrogen mass. From observations of the atomic hydrogen in NGC 4631, Rand (1994) finds a H I mass of the disk (excluding the Helium content) of $M_{\mathrm{HI}}=5 \times 10^{9} M_{\odot}$. We don't take into account here the mass of the various H I spurs detected by Rand (1994), which would add another $2.2 \times 10^{9} M_{\odot}$. The molecular mass found by Golla \& Wielebinski (1994) is $M_{\mathrm{H}_{2}}=1.2 \times 10^{9} M_{\odot}$, based on the galactic $\mathrm{CO}-\mathrm{H}_{2}$ conversion factor of $X=2.3 \times$ $10^{20} \mathrm{~cm}^{-2} /\left(\mathrm{K} \mathrm{km} \mathrm{s}^{-1}\right)$ (Strong et al. 1988). Although we know that $X$ may be variable and not necessarily valid for NGC 4631, the fraction of the molecular mass is rather small in this galaxy, thus we use $M_{\mathrm{H}}=6.2 \times 10^{9} M_{\odot}$. When we take this value for the hydrogen mass, assume a dust temperature of $18 \mathrm{~K}$ as determined above (note that at our observing wavelengths around $1 \mathrm{~mm}$ more than $99 \%$ of the dust emission comes from the cold component; see Fig. 4), and use Eq. (6) to calculate the absorption cross section for the whole galaxy, we get values of $\sigma_{0.87 \mathrm{~mm}}^{\mathrm{H}}=6.2 \pm 1.0 \times 10^{-27} \mathrm{~cm}^{2}$ and $\sigma_{1.23 \mathrm{~mm}}^{\mathrm{H}}=$ $6.4 \pm 0.8 \times 10^{-27} \mathrm{~cm}^{2}$ for both wavelengths.

In order to interpret these values, we use a formulation following Mezger et al. (1990),

$\sigma_{\lambda}^{\mathrm{H}}=C \lambda_{\mathrm{mm}}^{-2} b Z / Z_{\odot}$.

$C=7 \times 10^{-27} \mathrm{~cm}^{2}$ is the dust absorption cross section at $\lambda 1 \mathrm{~mm}$ following from the theoretical curves of Draine \& Lee (1984), $\lambda_{\mathrm{mm}}$ the wavelength in $\mathrm{mm}, Z$ the metallicity and $b$ an empirically determined factor which accounts for the differences between Drain \& Lee's grain mixtures and real grains. $b=1$ applies to dust in the diffuse interstellar H I gas, and since most gas in NGC 4631 is in atomic form, we will also use $b=1$ here. For solar metallicity we would thus expect values of 9.2 and $4.6 \times 10^{-27} \mathrm{~cm}^{2}$ for 0.87 and $1.23 \mathrm{~mm}$, respectively. At the latter wavelength, such a value has been measured for several nearby galaxies (e.g. Neininger et al. 1996; Dumke et al. 1997). Krügel \& Chini (1994) give a "standard" value which corresponds to $\sigma_{1.3 \mathrm{~mm}}^{\mathrm{H}}=5.0 \times 10^{-27} \mathrm{~cm}^{2}$ based on typical abundances of silicate and graphite in the ISM, which is in very good agreement with the values above and has been used successfully for fitting IR spectra over the whole spectrum from 1 to $1300 \mu \mathrm{m}$.

To compare our results with these values, we have to take the metallicity into account: Otte et al. (2002) measured the Nitrogen abundance in NGC 4631 and found an average metallicity of $Z / Z_{\odot} \sim 0.5$. This is also in agreement with the results from Vila-Costas \& Edmunds (1992) who determined metallicities and their gradients in Sd galaxies. With $Z=0.5 Z_{\odot}$, the observed absorption cross section $\sigma$ is a factor 1.5 higher than the predicted value for $\lambda 0.87 \mathrm{~mm}$, and even a factor 3 higher than the predicted value for $\lambda 1.23 \mathrm{~mm}$.

The dust absorption cross section can also be expressed by Hildebrand (1983)

$\sigma_{\lambda}^{\mathrm{H}}=\kappa_{\lambda} m_{\mathrm{H}} \frac{M_{\mathrm{d}}}{M_{\mathrm{g}}}$

with the dust absorption coefficient $\kappa_{\lambda}$ (which involves grain properties like size, density, and emissivity) and the dust-togas mass ratio $M_{\mathrm{d}} / M_{\mathrm{g}}$. The high values for $\sigma$ can thus be explained either with a high dust-to-gas ratio (which may be in contradiction to the low metallicity) or an increased absorption coefficient in the sub- $\mathrm{mm} / \mathrm{mm}$ regime, and therefore unusual optical properties of the dust grains in NGC 4631.

From Eq. (6) it is obvious that these high values for $\sigma$ are due to rather high flux densities. But when comparing the numbers for both wavelengths, the high value at $\lambda 1.23 \mathrm{~mm}$ can also be directly related to the unusual ratio between the two flux densities at our observing wavelengths. On the other hand, as shown in Fig. 3, we also see that the intensity ratio between these two wavelengths varies strongly along the galaxy's major axis. Therefore we also calculate the absorption cross section locally for selected areas along the major axis of NGC 4631, in order to trace local variations of the unusual dust properties found in the total spectrum.

The flux density per beam emitted by a cloud of gas and dust is (see Mezger et al. 1990)

$S_{\lambda}=\Omega_{\text {beam }} B_{\lambda}\left(T_{\mathrm{d}}\right)\left(1-\mathrm{e}^{-\tau_{\lambda}}\right)$

with $\tau_{\lambda}=\sigma_{\lambda}^{\mathrm{H}} N_{\mathrm{H}}$, where $\sigma_{\lambda}^{\mathrm{H}}$ is the dust absorption cross section per hydrogen atom and $N_{\mathrm{H}}$ the beam-averaged hydrogen column density. For $\tau_{\lambda} \ll 1$ we can transform Eq. (9) and express the cross section by

$\sigma_{\lambda}^{\mathrm{H}}=\frac{\lambda^{2}}{\Omega_{\text {beam }} 2 k T_{\mathrm{d}}} \frac{\left(\mathrm{e}^{x}-1\right)}{x} \frac{S_{\lambda}}{N_{\mathrm{H}}}$

with $x=h c / \lambda k T_{\mathrm{d}}$ and $N_{\mathrm{H}}=N(\mathrm{H} \mathrm{I})+2 N\left(\mathrm{H}_{2}\right)$.

We can use Eq. (10) to calculate the absorption cross section in various parts of NGC 4631. For this calculation we assume the temperature of the cold dust to decrease linearly from $20 \mathrm{~K}$ in the centre to $16 \mathrm{~K}$ in the outer parts, as found in the previous subsection. The result is shown in the upper panel of Fig. 5, which also shows (in the lower panel) the variation of the intensity ratio $I_{0.87 \mathrm{~mm}} / I_{1.23 \mathrm{~mm}}$ along the major axis.

Before we discuss these data, we average the results in various parts of the major axis in order to increase the $\mathrm{S} / \mathrm{N}$ : in the centre (strong $\mathrm{CO},|x|<1.5$ ), in the inner disk (weak $\mathrm{CO}$, $1.5 \leq|x|<4^{\prime}$ ), and in the outer disk (no detected CO, $|x| \geq 4^{\prime}$ ). 


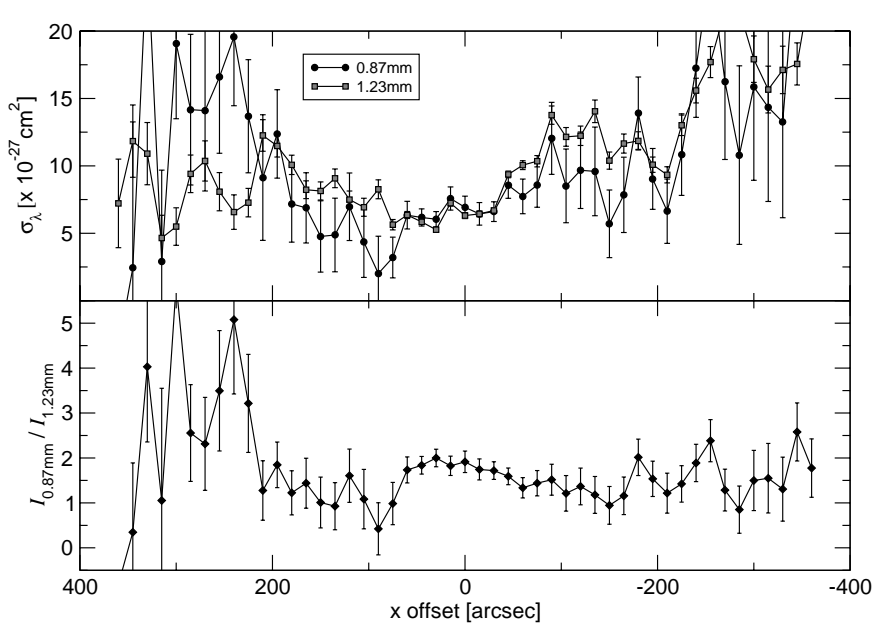

Fig. 5. Distribution of the intensity ratio of the two observing wavelengths (lower panel) and the absorption cross sections for a standard dust model (upper panel) along the major axis of NGC4631.

Table 3. Dust emission intensity ratios and absorption cross section in various regions along the major axis of NGC 4631.

\begin{tabular}{llll}
\hline \hline Position & $I_{0.87 \mathrm{~mm}} / I_{1.23 \mathrm{~mm}}$ & $\begin{array}{c}\sigma_{0.87 \mathrm{~mm}}^{\mathrm{H}} \\
{\left[\times 10^{-27} \mathrm{~cm}^{2}\right]}\end{array}$ \\
\hline$x \geq 4^{\prime}$ (east) & $2.8 \pm 0.6$ & $15 \pm 4$ & $8 \pm 2$ \\
$4^{\prime}>x \geq 1.5$ & $1.3 \pm 0.2$ & $7 \pm 2$ & $9 \pm 1$ \\
$1^{\prime} .5>x>-1^{\prime} .5$ & $1.7 \pm 0.1$ & $7 \pm 1$ & $7 \pm 1$ \\
$-1^{\prime} .5 \geq x>-4^{\prime}$ & $1.4 \pm 0.1$ & $9 \pm 2$ & $12 \pm 1$ \\
$x \leq-4^{\prime}$ (west) & $1.7 \pm 0.2$ & $19 \pm 3$ & $18 \pm 2$ \\
\hline
\end{tabular}

The obtained numbers for the dust intensity ratios and absorption cross sections are given in Table 3.

From these numbers, we can roughly distinguish three different situations. In the centre and inner disk, the absorption cross section at $\lambda 0.87 \mathrm{~mm}$ is only slightly higher than expected, whereas it is much higher at $\lambda 1.23 \mathrm{~mm}$. Also the intensity ratio is very low, especially in the areas of weak $\mathrm{CO}$ emission, where $\sigma_{1.23 \mathrm{~mm}}$ is even higher than $\sigma_{0.87 \mathrm{~mm}}$. In the outer parts however, where the optical image of NGC 4631 shows strong disturbances, the situation has changed. At the eastern edge of the disk, also $\sigma_{0.87 \mathrm{~mm}}^{\mathrm{HI}}$ is strongly enhanced, while the intensity ratio between both wavelengths is closer to the expected values. At the western edge, on the other hand, the intensity ratio is similar to the central area, but at both wavelengths the absorption cross section is increased by another factor 2 compared to the central area and inner disk.

To summarize the results of this subsection, we find that the dust absorption cross section in the sub- $\mathrm{mm} / \mathrm{mm}$ range is higher than what is predicted, and this result is especially significant in the outer disk $\left(|x|>4^{\prime}\right)$ of NGC 4631, where no molecular gas is detected. These areas are also strongly affected by the gravitational interaction of NGC 4631 with its neighbours. The optical image reveals strong disturbances of the disk, and also the footpoints of two H I spurs detected by Rand (1994) are located in these areas. A significant amount of molecular gas in these regions without corresponding $\mathrm{CO}$ emission could be responsible for the higher values of the absorption cross section at $\lambda 0.87 \mathrm{~mm}$, since this would result in an underestimate of $N_{\mathrm{H}}$ and therefore a too high value of $\sigma^{\mathrm{H}}$ (see Eq. (10)). This possibility is supported by results which suggest a higher value for the $\mathrm{CO}-\mathrm{H}_{2}$ conversion factor in the outer disks of galaxies and metal-poor environments - note that the metallicity in NGC 4631 decreases with increasing galactocentric radius (Otte et al. 2002). Nevertheless this possibility cannot explain why $\sigma_{1.23} \mathrm{~mm}$ is of the same order or even larger than $\sigma_{0.87 \mathrm{~mm}}$, or the observed excess of the dust emission at $\lambda 1.23 \mathrm{~mm}$. Another, more speculative scenario, would involve a different way of dust processing in those areas of NGC 4631 which are strongly affected by the interaction.

Therefore a reasonable dust model, whose properties can account for the mm excess detected above, must also be able to explain the enhanced values of $\sigma$ at sub- $\mathrm{mm} / \mathrm{mm}$ wavelengths.

\subsection{What is the origin of the $\mathrm{mm}$ excess?}

As mentioned above, the sub-mm/mm spectrum of NGC 4631 is too flat to be satisfactorily explained with a "standard" twocomponent dust model. However, several other dust models have been proposed in the past, including stochastic heating of very small particles, big grains at very low temperatures $(\ll 10 \mathrm{~K})$, or grains with different optical properties, like fractal, fluffy, or ice-coated grains. A good overview over several models is given by Reach et al. (1995) in an attempt to interpret COBE observations of our Galaxy. In the following we summarize the main points of these models in view of their application to the case of NGC 4631.

\subsubsection{Very cold big grains}

In most external galaxies which have been observed in the $\mathrm{mm}$ and sub-mm continuum the cold dust component has a temperature of 15-20 K. However, Siebenmorgen et al. (1999) have detected very cold dust in a sample of inactive spiral galaxies with an average temperature of $\sim 13 \mathrm{~K}$. These authors also discuss the possible existence of even colder dust and conclude that within the optical disk of a galaxy the dust cannot be colder than about $6 \mathrm{~K}$. If we want to explain the mm-excess of NGC 4631 with such a component of very cold grains, the most reasonable solution yields a temperature of $T_{\mathrm{vc}}=4 \mathrm{~K}$ for this temperature component, and the upper limit we find is $6 \mathrm{~K}$. Such a component has its maximum close to $\lambda \sim 1 \mathrm{~mm}$ and could therefore account for the very flat sub- $\mathrm{mm} / \mathrm{mm}$ part of the flux density spectrum. The fit to the data with temperatures of $50 \mathrm{~K}, 20 \mathrm{~K}$, and $4 \mathrm{~K}$ is shown in Fig. 6.

Such very cold dust could in principle exist in the form of self-shielded grains in very optically thick clouds with no intrinsic sources. In order to produce the measured flux density at $\lambda 1.23 \mathrm{~mm}$, the total dust mass needed is of the order $3-6 \times 10^{8} M_{\odot}$ (assuming an absorption coefficient as in the solar neighbourhood), most of which is at a temperature below $6 \mathrm{~K}$. With the gas mass estimated from the $\mathrm{CO}$ and $\mathrm{HI}$ data $\left(8.5 \times 10^{9} M_{\odot}\right.$ including the helium content) this leads to 



Fig. 6. Two alternative possibilities to fit the FIR-to-mm spectrum of NGC 4631. The left figure shows a possible interpretation including a component of very cold dust. The right figure shows a model fit with an increased abundance of very small grains. Both possibilities are discussed in the text.

a gas-to-dust ratio of 15-30, far below values in other galaxies or the Milky Way. While this low ratio could explain the high value we found for the dust absorption cross section $\sigma$ (see Eq. (8)), it is in contradiction to the low metallicities in NGC $4631\left(Z / Z_{\odot} \sim 0.5\right.$ on average) which were found recently by Otte et al. (2002).

On the other hand, Krügel \& Siebenmorgen (1994) have shown that in cold dense clouds the dust grains may coagulate to ice-coated very big grains (up to a radius $a \sim 100 \mu \mathrm{m}$ ), for which the absorption coefficient can be enhanced by a factor of eight relative to the diffuse ISM. If a large amount of cold dust existed in this form, the dust emission could be explained with gas-to-dust ratios similar to values in the local ISM.

However, in order to keep a large amount of dust at temperatures below $6 \mathrm{~K}$, compared to the cold component with 15$20 \mathrm{~K}$, the interstellar radiation field must be significantly attenuated. The necessary extinction is of the order $A_{V}=15-50$ (depending on grain type), corresponding to a gas column density of $N_{\mathrm{H}} \sim 10^{22}$ or more (see Reach et al. 1995, and references therein). Figure 3 shows that values of $N_{\mathrm{H}} \sim 10^{22}$ for the total hydrogen column density are reached only in the inner $3^{\prime}$ of NGC 4631, around the maxima of the CO distribution (assuming a standard $\mathrm{CO}-\mathrm{H}_{2}$ conversion factor). While we would expect radiation-shielded cold dust to exist at places of dense molecular gas, these regions are also places of strong star formation. In addition, the mm excess is present over the whole disk of NGC 4631, and especially at radii of $1.5-3^{\prime}$, where the ratio $I_{0.87 \mathrm{~mm}} / I_{1.23 \mathrm{~mm}}$ is smallest (see Fig. 5). These areas are located well beyond the molecular ring.

On the other hand, Galliano et al. (2003) consistently explained the mm-excess in NGC 1569 with very cold grains deeply embedded in clumps of big grains and primarily heated by the FIR emission of the latter. This would require a very clumpy medium and small filling factors of the cold gas and dust.

\subsubsection{Very small grains}

A different model which was successfully applied to the absorption and emission data from our galaxy over a large wavelength range was presented by Désert et al. (1990). This model included (besides big grains, which radiate mainly at $\lambda>$ $70 \mu \mathrm{m}$ ) a component of amorphous, very small grains (VSGs), which are the main contributor to the IR emission between 10 and $70 \mu \mathrm{m}$, and of Polycyclic Aromatic Hydrocarbons (PAHs), which radiate shortwards of $10 \mu \mathrm{m}$. The VSGs, which are about $1-20 \mathrm{~nm}$ in size, are so small that they are heated to nonequilibrium temperatures by absorption of a single photon and thus show a fluctuating temperature distribution. They also have a much broader FIR emission spectrum compared to big grains, and an absorption coefficient proportional to $\lambda^{-1}$, i.e. $\beta=1$ can be assumed for this component (Seki \& Yamamoto 1980).

Besides our own galaxy, also the FIR-to-mm spectrum of the dwarf galaxy NGC 1569 was succesfully fitted with this dust model (Lisenfeld et al. 2002), although with a stronger ambient radiation field and a different grain composition (increased abundance of VSGs and absence of PAHs) than the solar neighbourhood.

We tried to fit the FIR to mm spectrum of NGC 4631 with the dust model of Désert et al. (1990); the result is shown in Fig. 6. For this fit we neglected the PAH component which radiates at wavelengths smaller than $25 \mu \mathrm{m}$. In order to fit the data, we have to assume an interstellar radiation field and dust composition different from the solar neighbourhood. From the FUV data published by Smith et al. (2001), we estimate an average ISRF within NGC 4631 at $\lambda 1000 \AA$ of about $6 \times 10^{-2} \mathrm{erg} \mathrm{cm}^{-2} \mathrm{~s}^{-1} \mu \mathrm{m}^{-1}$, which is four times the local ISRF as given in Mezger et al. (1982). Compared to big grains, which have an average temperature of $\sim 22 \mathrm{~K}$ in this model, VSGs are overabundant by a factor of 4 in order to account for the small $S(0.87 \mathrm{~mm}) / S(1.23 \mathrm{~mm})$ ratio with the shallower VSG spectrum in the FIR. In addition, we assume that the VSGs have sizes up to $20 \mathrm{~nm}$, compared to $15 \mathrm{~nm}$ as in the Galaxy. This latter assumption does not necessarily change the physical properties of the VSGs, like surface-to-volume ratio and heat capacity (Kamijo et al. 1975; Stephens \& Russell 1979).

The application of this model to NGC 4631 has its drawbacks as well. The estimated dust mass for the big grain component, assuming standard optical properties, is $1.1 \times 10^{7} M_{\odot}$, while the VSGs add up to $0.3 \times 10^{7} M_{\odot}$. The resulting gasto-dust ratio of $\sim 600$ is similar to values in extremely metalpoor environments, and therefore somewhat too high for the metallicities measured in NGC 4631. Another critical point is the ISRF of 4 times solar which is required for a reasonable fit. While this value seems appropriate in the inner part of NGC 4631, it is unclear if and how such a radiation field can be maintained in the outer disk, where the measured absorption cross sections are highest. Besides these physical concerns, the model fit to the data resulted in a $\chi^{2}$ much worse than the model including a component of very cold grains.

\subsubsection{Grains with unusual optical properties}

Fractal grains have an enhanced efficiency at sub-mm and mmwavelengths compared to the optical (e.g. Wright 1993). Thus a population of fractal grains may exist which show rapid temperature fluctuations (of a few K), but spend most of the time 
at very low temperatures due to high FIR-to-mm emissivity. While the exact properties of this type of grains are not yet well established, it is known that a much smaller amount of these grains is needed (compared to compact spherical grains) in order to produce a comparable amount of emission, hereby explaining the large values for $\sigma$ found in the previous subsection. And while the temperature spectrum of these grains may well account for the measured mm flux density, their mass plays only a minor role in the total dust mass of the galaxy. Thus the gas-to-dust ratio could have a reasonable value and agree with the low metallicity inferred from the Nitrogen abundance.

If such a component exists, we have to ask why it is detected only in a few objects. It may be responsible for part of the mm radiation of the Milky Way (Reach et al. 1995) and the dwarf starburst galaxy NGC 1569 (Galliano et al. 2003; Lisenfeld et al. 2002), although the latter authors explained this emission with an overabundance of VSGs. Many other galaxies do not show any excess in the mm range, but why should this fractal grain population exist in these apparently very different objects, but not in many other galaxies of various types? We can argue that sub-mm continuum observations have just reached the required sensitivity to detect this effect, and for many objects only data in the $\mathrm{mm}$ or in the sub-mm regime exist, while our results show that it is necessary to observe at both wavelengths to investigate the dust properties of these objects.

After all we should note that in principle strong variations in strength and colour of the interstellar radiation field may also cause a broadening of the dust spectrum, hereby potentially flattening the spectrum in the sub- $\mathrm{mm} / \mathrm{mm}$ range. However, shortwards of $\lambda 0.87 \mathrm{~mm}$ the spectrum is not flattened and consistent with a dust absorption coefficient $\propto \lambda^{-2}$. Furthermore, the discovered excess in the $\mathrm{mm}$ is quite significant, and with the complexity of the ISM also in normal galaxies the question arises why the temperature distribution of dust should be so much narrower in most other galaxies observed up to date, despite their varying classification and star formation properties.

\section{Summary and outlook}

We have observed the nearby interacting galaxy NGC 4631 in the radio continuum emission at $\lambda \lambda 0.87 \mathrm{~mm}$ and $1.23 \mathrm{~mm}$. The emission is concentrated on the galactic plane, although some halo emission could also be detected, especially at $1.23 \mathrm{~mm}$ where intergalactic cold dust seems to follow the previously detected H I spurs. The two maxima visible in both maps, located in the plane symmetrically around the nucleus at a radius of about $30^{\prime \prime}$, resemble a ring-like structure, similar to the distribution of the $\mathrm{CO}$ molecular line emission. In the outer disk, where no $\mathrm{CO}$ is detected, the $\mathrm{mm} / \mathrm{sub}-\mathrm{mm}$ emission follows the $\mathrm{H}$ I distribution, similar to other edge-on galaxies.

After subtracting non-dust contributions (line, synchrotron, and free-free emission) from the bolometer flux, the remaining flux densities of $S_{0.87 \mathrm{~mm}}=3.41 \pm 0.58 \mathrm{mJy}$ and $S_{1.23 \mathrm{~mm}}=$ $2.04 \pm 0.23 \mathrm{mJy}$ can be attributed to the thermal emission of dust. In order to estimate the temperature of this dust, we fitted a two-component modified Planck spectrum to the FIR-to-mm data and found a temperature of $50 \mathrm{~K}$ for the warm component and of $18 \mathrm{~K}$ for the cold component, which is responsible for most of the emission at $\lambda>200 \mu \mathrm{m}$.

This two-component model suffers from the fact that the observed dust spectrum is too flat in the sub- $\mathrm{mm} / \mathrm{mm}$ range. Furthermore it leads to absorption cross sections too high compared with theoretical expectations or previous results on other galaxies, especially in the outer part of the disk which are disturbed because of gravitational interaction.

We suggest several possibilites to resolve these inconsistencies, the most likely being grains with unusual optical properties which can account for the measured mm-excess as well as the high absorption cross sections.

These observations have shown that results of $\mathrm{mm}$ and sub$\mathrm{mm}$ observations have to be combined to investigate the cold dust component in external galaxies.

Acknowledgements. We thank the staff of the HHT and the 30-m telescope for their excellent support, and O. Löhmer and N. Neininger for their collaboration during the observations. Further we would like to thank E. Krügel, U. Lisenfeld, C. Popescu, and R. Tuffs for discussions and comments which helped to improve the paper.

\section{References}

Alton, P. B., Bianchi, S., Rand, R. J., et al. 1998, ApJ, 507, L125

Alton, P. B., Davies, J. I., \& Bianchi, S. 1999, A\&A, 343, 51

Andriesse, C. D. 1974, A\&A, 37, 257

Baars, J. W. M., Martin, R. N., Mangum, J. G., McMullin, J. P., \& Peters, W. L. 1999, PASP, 111, 627

Bendo, G. J., Joseph, R. D., Wells, M., et al. 2002, AJ, 123, 3067

Bendo, G. J., Joseph, R. D., Wells, M., et al. 2003, AJ, 125, 2361

Bicay, M. D., Helou, G., \& Condon, J. J. 1989, ApJ, 338, L53

Braine, J., Krügel, E., Sievers, A., \& Wielebinski, R. 1995, A\&A, 295, L55

Braine, J., Guélin, M., Dumke, M., et al. 1997, A\&A, 326, 963

Chini, R., Krügel, E., Lemke, R., \& Ward-Thompson, D. 1995, A\&A, 295, 317

de Vaucouleurs, G., de Vaucouleurs, A., Corwin, H. G. Jr., et al. 1991, Third reference catalogue of bright galaxies (New York: SpringerVerlag)

Désert, F.-X., Boulanger, F., \& Puget, J. L. 1990, A\&A, 237, 215

Devereux, N. A., \& Young, J. S. 1990, ApJ, 359, 42

Draine, B. T., \& Lee, H. M. 1984, ApJ, 285, 89

Dumke, M., Braine, J., Krause, M., et al. 1997, A\&A, 325, 124

Dumke, M., Nieten, Ch., Thuma, G., Wielebinski, R., \& Walsh, W. 2001, A\&A, 373, 853

Ekers, R. D., \& Sancisi, R. 1977, A\&A, 54, 973

Galliano, F., Madden, S. C., Jones, A. P., et al. 2003, A\&A, 407, 159

Golla, G., \& Wielebinski, R. 1994, A\&A, 286, 733

Haynes, M. P., Giovanelli, R., \& Roberts, M. S. 1979, ApJ, 229, 83

Hildebrand, R. H. 1983, QJRAS, 24, 267

Kamijo, F., Nakada, Y., Iguchi, T., Fujimoto, M.-K., \& Takada, M. 1975, Icarus, 26, 102

Krügel, E., \& Chini, R. 1994, A\&A, 287, 947

Krügel, E., \& Siebenmorgen, R. 1994, A\&A, 288, 929

Lisenfeld, U., Isaak, K. G., \& Hills, R. 2000, MNRAS, 312, 433

Lisenfeld, U., Israel, F. P., Stil, J. M., \& Sievers, A. 2002, A\&A, 382, 860

Martin, C., \& Kern, B. 2001, ApJ, 555, 258

Mezger, P. G., Mathis, J. S., \& Panagia, N. 1982, A\&A, 105, 372

Mezger, P. G., Wink, J. E., \& Zylka, R. 1990, A\&A, 228, 95 
Neininger, N., \& Dumke, M. 1999, Proc. Natl. Acad. Sci. USA, 96, Siebenmorgen, R., Krügel, E., \& Chini, R. 1999, A\&A, 351,495 5360

Neininger, N., Guélin, M., García-Burillo, S., Zylka, R., \& Wielebinski, R. 1996, A\&A, 310, 725

Smith, A. M., Collins, N. R., Waller, W. H., et al. 2001, ApJ, 546, 829

Stevens, J. A., \& Gear, W. K. 2000, MNRAS, 312, L5

Niklas, S., Klein, U., Braine, J., \& Wielebinski, R. 1995, A\&AS, 114, Strong, A. W., Bloemen, J. B. G. M., Dame, T. M., et al. 1988, A\&A, 21

Niklas, S., Klein, U., \& Wielebinski, R. 1997, A\&A, 322, 19

Otte, B., Gallagher III, J. S., \& Reynolds, R. J. 2002, ApJ, 572, 823

Popescu, C. C., Tuffs, R. J., Völk, H. J., Pierini, D., \& Madore, B. F. 2002, ApJ, 567, 221

Rand, R. J. 1994, A\&A, 285, 833

Rand, R. J. 2000, ApJ, 533, 663

Reach, W. T., Dwek, E., Fixsen, D. J., et al. 1995, ApJ, 451, 188

Reuter, H.-P., Krause, M., Wielebinski, R., \& Lesch, H. 1991, A\&A, 248,12

Rice, W., Lonsdale, C. J., Soifer, B. T., et al. 1988, ApJS, 68, 91

Seki, J., \& Yamamoto, T. 1980, Ap\&SS, 72, 79 